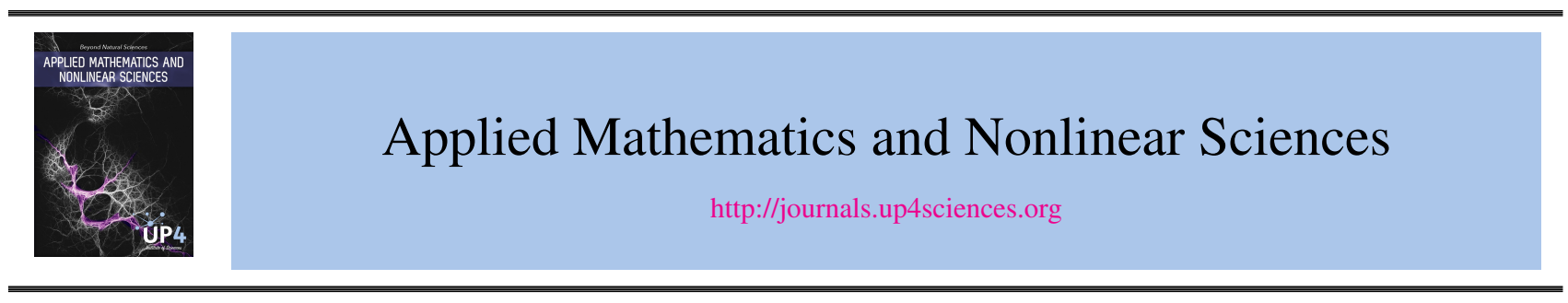

\title{
Homotopy analysis method for the solution of lubrication of a long porous slider
}

\author{
Vishwanath B.Awati ${ }^{\dagger}$, Manjunath Jyoti. \\ Department of Mathematics, Rani Channamma University, Belagavi, 591156 \\ India
}

Submission Info

Communicated by Juan L.G. Guirao

Received 5th June 2016

Accepted 1st November 2016

Available online 1st November 2016

\begin{abstract}
In this article, the lubrication of a long porous slider in which the fluid is injected into the porous bottom is considered. The similarity transformations reduce the governing problem of Navier-Stokes equations to coupled nonlinear ordinary differential equations which are solved by HAM. Solutions are obtained for much larger values of Reynolds number compared to analytical and numerical methods. The results comprise good agreement between approximate and numerical solutions. HAM gives rapid convergent series solutions which show that this method is efficient, accurate and has advantages over other methods. Further, homotopy-pade' technique is used to accelerate the convergence of series solution.
\end{abstract}

Keywords: Porous slider; Reynolds number; HAM; Homotopy-Pade' approximations.

AMS 2010 codes: 76-xx, 76Sxx, 34-xx.

\section{Introduction}

The problem of a long porous slider in which the fluid is passed through the porous bottom is considered. In general, the sliding friction is highly reduced by injecting a fluid between two solid surfaces moving relative to each other. Sliding friction means the force that is required to keep a surface sliding along other surface. The porous sliders have an important role in fluid cushioned moving pads and practical applications include hydrostatic thrust bearings and air cushioned vehicles. Many authors have worked on porous slider which includes the porous circular slider [1], the porous flat slider [2,3], and the porous elliptic slider [4,5]. Skalak and Wang [2] discussed the solution for the porous flat slider by means of inverse method. The laminar flow between porous plates/disks has been studied by many researchers [6]- [11]. In most of the flow problems which are two-dimensional or axisymmetric, those are simplified forms of the real situations. To gain insight into the

$\dagger$ Corresponding author.

Email address: awati_vb@yahoo.com

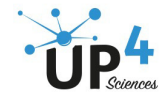


real situation, an attempt is made to analyze the three-dimensional problem. Recently, Awati et.al [12] discussed the solution of MHD flow of viscous fluid between two parallel porous plates using CESS and HAM.

In this paper we investigate the three dimensional flow problems using the homotopy analysis method [13][15]. This method is a semi-analytical technique which is quite useful and interesting for the solution of highly nonlinear ordinary and partial differential equations. The method constructs a homotopy between the nonlinear differential operator and an auxiliary linear operator. The HAM involves convergence control parameter $\hbar$, which adjust the convergence region and the rate of approximation of the solution by series. From all the above, the HAM has an excellent flexibility and generality over all other analytical and approximation methods and also it is easy to use.

The paper is structured as follows. Section 1 describes the introduction; section 2 develops the mathematical formulation of the proposed problem with relevant boundary conditions. Section 3 gives the semi-analytical solution of the proposed problem using the Homotopy analysis method. Section 4 presents results and discussion; section 5 is about the conclusion.

\section{Mathematical formulation}

Let us consider a long porous slider of dimension $L_{1}$ and $L_{2}$ (Fig. 1 (a) and (b)) in which the fluid is passed through the porous bottom of slider with velocity $W$ such that a small gap is created and width is $d$. The porous slider moves laterally with velocity $U$ and longitudinally with velocity $V$ along the $x$ and $y$-directions respectively which is shown in Fig. 1 (a).

(a)

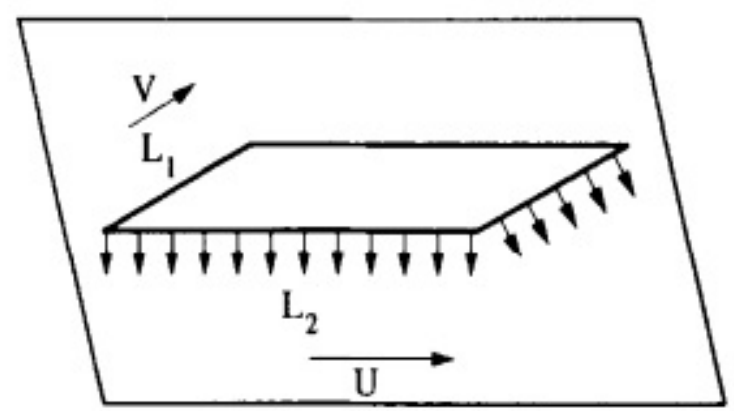

(b)

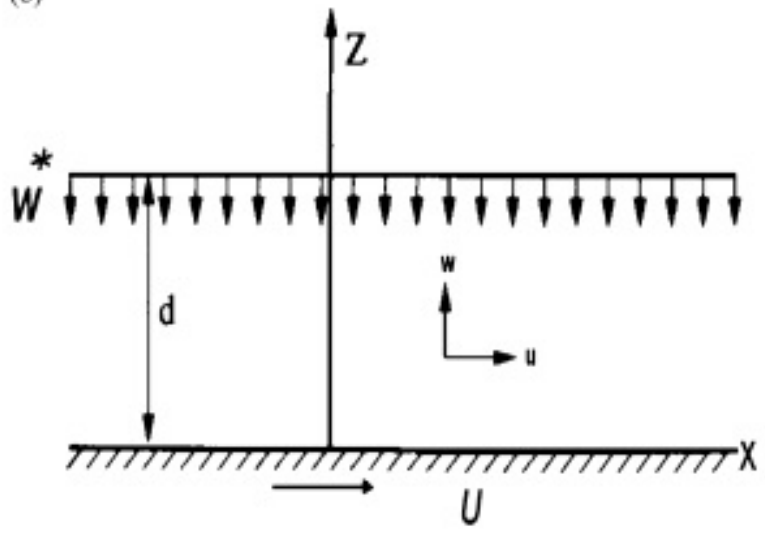

Fig. 1 (a) Moving long porous slider; (b) Coordinate system.

Assume that $L_{2} \geq L_{1} \geq d$ such that the end effects can be neglected. Let $u, v$ and $w$ are the velocity components of the fluid along $x, y$ and $z$ directions respectively. The basic equations for the flow problem are Navier-Stokes equations and continuity equations [2].

$$
\begin{gathered}
(q \cdot \nabla) q=-\frac{1}{\rho} \nabla p+\gamma \nabla^{2} q \\
\nabla \cdot q=0
\end{gathered}
$$

where $q=(u, v, w), \nabla=\left(\frac{\partial}{\partial x}, \frac{\partial}{\partial y}, \frac{\partial}{\partial z}\right)$ is the del operator, $p$ is the pressure of the fluid and $\gamma$ is the kinematic 
viscosity. The appropriate boundary conditions of the flow problem becomes

$$
\left.\begin{array}{ccc}
u=U, v=V, w=0 & \text { at } & z=0 \\
u=0, v=0, w=-W & \text { at } & z=d
\end{array}\right\}
$$

Equations (1)-(3) admits a similarity transformation [2] such as

$$
u=U f(\eta)+\frac{W x}{d} h^{\prime}(\eta), \quad v=V g(\eta), \quad w=-W h(\eta)
$$

where $\eta=z / d$ is a similarity variable and the above transformations reduces the NS equations to a set of coupled nonlinear ordinary differential equations

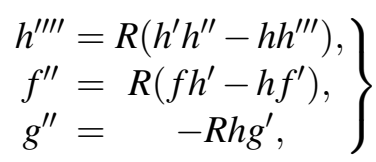

where $R=\frac{W d}{\gamma}$ is the cross flow Reynolds number. The corresponding boundary conditions takes the form

$$
\left.\begin{array}{l}
h(0)=0=h^{\prime}(0), f(0)=1=g(0), \\
h(1)=1=h^{\prime}(1), f(1)=0=g(1) .
\end{array}\right\}
$$

\section{Homotopy analysis method}

We employ the HAM for the solution of Eqn. (5) subjected to the boundary conditions (6). We choose the initial guesses which satisfies the boundary conditions automatically and auxiliary linear operators for the functions $h, f$ and $g$

$$
h_{0}(\eta)=3 \eta^{2}-2 \eta^{3}, \quad f_{0}(\eta)=1-(\eta), \quad g_{0}(\eta)=1-(\eta)
$$

and

$$
L[h]=h^{\prime \prime \prime \prime}, \quad L[f]=f^{\prime \prime}, \quad L[g]=g^{\prime \prime} .
$$

Here the above linear operator satisfies

$$
L_{h}\left[C_{1} \eta^{3}+C_{2} \eta^{2}+C_{3} \eta+C_{4}\right]=0, \quad L_{f}\left[C_{5} \eta+C_{6}\right]=0, \quad L_{g}\left[C_{7} \eta+C_{8}\right]=0,
$$

where $C_{i}: i=1-8$ are arbitrary constants to be determined later.

\subsection{Zeroth-order deformation problem}

If $q \in[0,1]$ then the zeroth order deformation problem can be constructed as

$$
\left.\begin{array}{rl}
(1-q) L_{h}\left[h(\eta, q)-h_{0}(\eta)\right] & =q \hbar_{h} \aleph_{h}[h(\eta, q), f(\eta, q), g(\eta, q)], \\
(1-q) L_{f}\left[f(\eta, q)-f_{0}(\eta)\right] & =q \hbar_{f} \aleph_{f}[h(\eta, q), f(\eta, q), g(\eta, q)], \\
(1-q) L_{g}\left[g(\eta, q)-g_{0}(\eta)\right] & =q \hbar_{g} \aleph_{g}[h(\eta, q), f(\eta, q), g(\eta, q)] .
\end{array}\right\}
$$

The boundary conditions becomes

$$
\left.\begin{array}{l}
h(0, q)=0, h(1, q)=1, h^{\prime}(0, q)=0, h^{\prime}(1, q)=0, \\
f(0, q)=0, f(1, q)=0, g(0, q)=1, g(1, q)=0,
\end{array}\right\}
$$

where $q \in[0,1]$ is an embedding parameter. Here $\hbar_{h}, \hbar_{f}$ and $\hbar_{g}$ are non-zero auxiliary parameters. Further $\boldsymbol{\aleph}$ is the non-linear differential operator and is given by

$$
\begin{aligned}
& \aleph_{h}[h(\eta, q)] \quad=\frac{\partial^{4} h(\eta, q)}{\partial \eta^{4}}-R\left(\frac{\partial h(\eta, q)}{\partial \eta} \frac{\partial^{2} h(\eta, q)}{\partial \eta^{2}}-h(\eta, q) \frac{\partial^{3} h(\eta, q)}{\partial \eta^{3}}\right), \\
& \left.\aleph_{f}[h(\eta, q), f(\eta, q)]=\frac{\partial^{2} f(\eta, q)}{\partial \eta^{2}}-R\left(f(\eta, q) \frac{\partial h(\eta, q)}{\partial \eta}-h(\eta, q) \frac{\partial f(\eta, q)}{\partial \eta}\right),\right\} \\
& \aleph_{g}[h(\eta, q), g(\eta, q)]=\quad \frac{\partial^{2} g(\eta, q)}{\partial \eta^{2}}+R h(\eta, q) \frac{\partial g(\eta, q)}{\partial \eta} \text {. }
\end{aligned}
$$


For $q=0$ and $q=1$, Eqn. (7) have the solutions

$$
\left.\begin{array}{ll}
h(\eta, 0)=h_{0}(\eta) & h(\eta, 1)=h(\eta), \\
f(\eta, 0)=f_{0}(\eta) & f(\eta, 1)=f(\eta), \\
g(\eta, 0)=g_{0}(\eta) & g(\eta, 1)=g(\eta) .
\end{array}\right\}
$$

As $q$ vary from 0 to $1, h(\eta, q), f(\eta, q), g(\eta, q)$ also vary from the initial guesses $h_{0}(\eta), f_{0}(\eta), g_{0}(\eta)$ to the final solutions $h(\eta), f(\eta), g(\eta)$. With the help of Taylor's theorem, Eqn. (8) can be written as

$$
\left.\begin{array}{l}
h(\eta, q)=h_{0}(\eta)+\sum_{m=1}^{\infty} h_{m}(\eta) q^{m}, \\
f(\eta, q)=f_{0}(\eta)+\sum_{m=1}^{\infty} f_{m}(\eta) q^{m}, \\
g(\eta, q)=g_{0}(\eta)+\sum_{m=1}^{\infty} g_{m}(\eta) q^{m},
\end{array}\right\}
$$

where $h_{m}(\eta)=\left.\frac{1}{m !} \frac{\partial^{m} h(\eta, q)}{\partial q^{m}}\right|_{q=0}, f_{m}(\eta)=\left.\frac{1}{m !} \frac{\partial^{m} f(\eta, q)}{\partial q^{m}}\right|_{q=0}, g_{m}(\eta)=\left.\frac{1}{m !} \frac{\partial^{m} g(\eta, q)}{\partial q^{m}}\right|_{q=0}$. The convergence of the above series (14) depends on the auxiliary parameters $\hbar_{h}, \hbar_{f}$ and $\hbar_{g}$. In order to select the values of $\hbar_{h}, \hbar_{f}$ and $\hbar_{g}$ in such a way that the series (14) is convergent at $q=1$, we have

$$
\left.\begin{array}{l}
h(\eta, q)=h_{0}(\eta)+\sum_{m=1}^{\infty} h_{m}(\eta), \\
f(\eta, q)=f_{0}(\eta)+\sum_{m=1}^{\infty} f_{m}(\eta), \\
g(\eta, q)=g_{0}(\eta)+\sum_{m=1}^{\infty} g_{m}(\eta) .
\end{array}\right\}
$$

\section{2 mth-order deformation problem}

Differentiating the zeroth order deformation problem (7) $m$ times with respect to the embedding parameter $q$ and then dividing by $m$ !, finally setting $q=0$. The resulting mth-order deformation problem becomes

$$
\left.\begin{array}{l}
L_{h}\left[h_{m}(\eta)-\chi_{m} h_{m-1}(\eta)\right]=\hbar_{h} \Re_{m}^{h}(\eta), \\
L_{f}\left[f_{m}(\eta)-\chi_{m} f_{m-1}(\eta)\right]=\hbar_{f} \Re_{m}^{f}(\eta), \\
L_{g}\left[g_{m}(\eta)-\chi_{m} g_{m-1}(\eta)\right]=\hbar_{g} \Re_{m}^{g}(\eta) .
\end{array}\right\}
$$

The homogeneous boundary conditions are

$$
\left.\begin{array}{c}
h_{m}(0, q)=0, h_{m}(1, q)=0, h_{m}^{\prime}(0, q)=0, h_{m}^{\prime}(1, q)=0, \\
f_{m}(0, q)=0, f_{m}(1, q)=0, g_{m}(0, q)=0, g_{m}(1, q)=0,
\end{array}\right\}
$$

where

$$
\begin{aligned}
& \Re_{m}^{h}(\eta)=h_{m-1}^{\prime \prime \prime \prime}+R \sum_{n=0}^{m-1}\left[h_{n} h_{m-1-n}^{\prime \prime \prime}-h_{n}^{\prime} h_{m-1-n}^{\prime \prime}\right],
\end{aligned}
$$

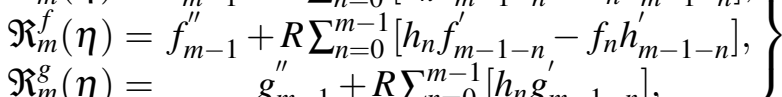

and

$$
\chi_{m}=\left\{\begin{array}{l}
0, m \leq 1 ; \\
1, m>1 ;
\end{array}\right.
$$

We use Mathematica to solve the linear system of equations (11) with the appropriate homogeneous boundary conditions (12) up to first few orders of approximations for the series $h, f$ and $g$

$$
\begin{aligned}
h_{1} & =\hbar_{h} R\left(-\frac{8}{35} \eta^{2}+\frac{27}{70} \eta^{3}-\frac{3}{10} \eta^{5}+\frac{1}{5} \eta^{6}-\frac{2}{35} \eta^{7}\right), \\
h_{2} & =\frac{1}{646800} \hbar_{h} R(-1+\eta)^{2}\left(-9240\left(16+5 \eta-6 \eta^{2}+4 \eta^{3}\right)+\hbar_{h}\left(-9240\left(16+5 \eta-6 \eta^{2}+4 \eta^{3}\right)+R(-761\right.\right. \\
& \left.\left.\left.-7380 \eta-13999 \eta^{3}-2905 \eta^{4}-504 \eta^{5}-1568 \eta^{6}+448 \eta^{7}\right)\right)\right), \\
f_{1} & =\hbar_{f} R\left(\frac{9}{20} \eta-\eta^{3}+\frac{3}{4} \eta^{4}-\frac{1}{5} \eta^{5},\right.
\end{aligned}
$$




$$
\begin{aligned}
f_{2} & =\frac{1}{25200} \hbar_{f} R \eta\left(-6(-1+\eta)\left(210\left(9+9 \eta-11 \eta^{2}+4 \eta^{3}\right)+\hbar_{h} R\left(-52-52 \eta+268 \eta^{2}-217 \eta^{3}-55 \eta^{4}\right.\right.\right. \\
& \left.\left.+155 \eta^{5}-85 \eta^{6}+20 \eta^{7}\right)\right)+\hbar_{f}\left(-1260\left(-9+20 \eta^{2}-15 \eta^{3}+4 \eta^{4}\right)+R\left(1592-2835 \eta^{3}+2268 \eta^{4}\right.\right. \\
& \left.\left.\left.-2520 \eta^{5}-2700 \eta^{6}-1485 \eta^{7}+280 \eta^{8}\right)\right)\right), \\
g_{1} & =\hbar_{g} R\left(\frac{3}{20} \eta-\frac{1}{4} \eta^{4}+\frac{1}{10} \eta^{5},\right. \\
g_{2} & =-\frac{1}{25200} \hbar_{g} R \eta\left(-2\left(630\left(3-5 \eta^{3}+2 \eta^{4}\right)+\hbar_{h} R\left(-52+240 \eta^{3}-243 \eta^{4}+90 \eta^{6}-45 \eta^{7}+10 \eta^{8}\right)\right)\right. \\
& \left.+\hbar_{g}\left(-1260\left(3-5 \eta^{3}+2 \eta^{4}\right)+R\left(-8-945 \eta^{3}+378 \eta^{4}+1800 \eta^{6}-1575 \eta^{7}+350 \eta^{8}\right)\right)\right) .
\end{aligned}
$$

\subsection{Convergence of HAM}

The analytic expressions of $h, f$ and $g$ in terms of series are given in Eqn. (10) contains the auxiliary parameters and the convergence of the series strictly depends upon the value of parameters $\hbar_{h}, \hbar_{f}$ and $\hbar_{g}$ which are called as convergence control parameters. These parameter plays a vital role in determining the convergence region and the rate of approximation for the HAM. For this purpose, we have drawn the line segment of the $\hbar$ curves parallel to $\eta$-axis. Fig. 2 shows the $\hbar$ curves for the series $h^{\prime \prime \prime}(0), f^{\prime}(0)$ and $g^{\prime}(0)$ for the 15 th order of approximations. It is clearly indicates that the admissible ranges of $\hbar_{h}, \hbar_{f}$ and $\hbar_{g}$ are $-1.5 \leq \hbar_{h} \leq-0.5,-1.5 \leq$ $\hbar_{f} \leq-0.25$ and $-2 \leq \hbar_{g} \leq-0.25$ respectively for $R=1$. Our calculation shows that the all the three series converges in the whole region of $0 \leq \eta \leq 1$ when $\hbar_{h}=\hbar_{f}=\hbar_{g}=-1$, other than this $\hbar$ values the results may diverge or converges slowly.

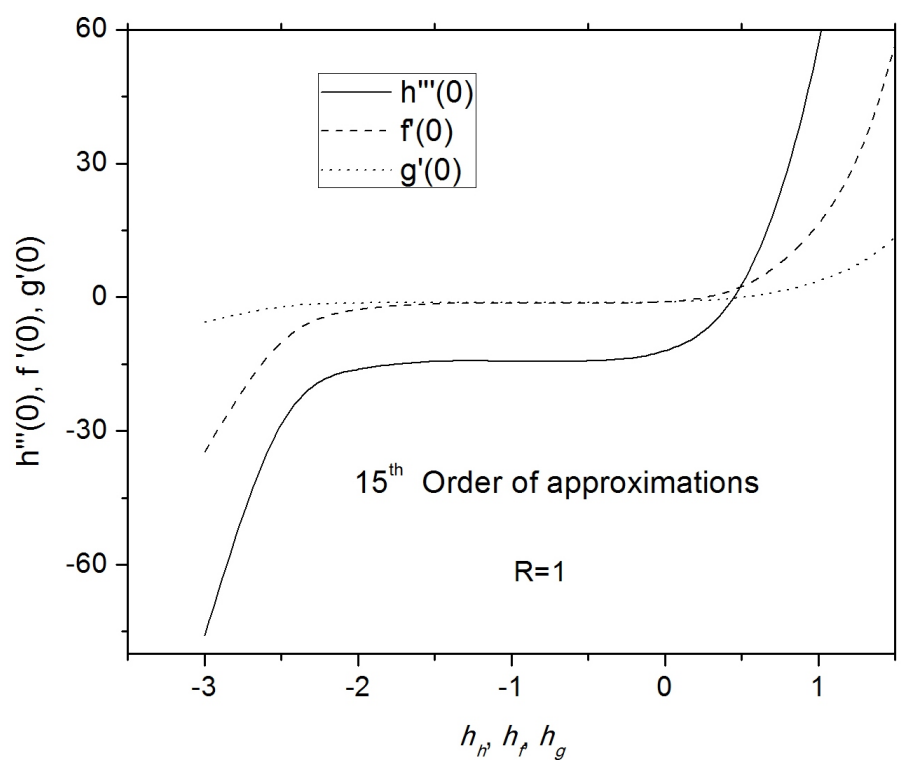

Fig. $2 \hbar$ curves for the series $h^{\prime \prime \prime}(0), f^{\prime}(0)$ and $g^{\prime}(0)$ for $R=1$.

\section{Results and Discussion}

The equation of motion for the lubrication of a long porous slider is governed by coupled nonlinear ODEs (5) together with the boundary conditions (6) is solved by HAM. This section discusses the effects of Reynolds number on velocity profiles, skin friction and lifts and drag coefficients. Table 1 shows the convergence of HAM solutions for different order of approximations; it is observed that the series solution converges at the thirty fifth orders of approximations. 
Table 1 Convergence of HAM solutions for different order of approximations for $R=10$ when $\hbar_{h}=\hbar_{f}=\hbar_{g}=-1$.

\begin{tabular}{|c|c|c|c|}
\hline order of approximation & $h^{\prime \prime \prime}(0)$ & $f^{\prime}(0)$ & $g^{\prime}(0)$ \\
\hline 5 & -37.8737 & -3.51618 & -2.37722 \\
\hline 10 & -38.1065 & -3.45718 & -2.41558 \\
\hline 15 & -38.1026 & -3.46067 & -2.41123 \\
\hline 20 & -38.1024 & -3.4605 & -2.40905 \\
\hline 25 & -38.1024 & -3.46051 & -2.40864 \\
\hline 30 & -38.1024 & -3.46051 & -2.40861 \\
\hline 35 & -38.1024 & -3.46051 & -2.40862 \\
\hline 40 & -38.1024 & -3.46051 & -2.40862 \\
\hline
\end{tabular}

Further, the homotopy-pade technique is used to accelerate the convergence of series solution, which is more efficient than the traditional Pade technique. The $[m, m]$ homotopy-pade approximations of $h^{\prime \prime \prime}(0), f^{\prime}(0)$ and $g^{\prime}(0)$ are given in Table 2 .

Table 2 The $[m, m]$ homotopy-Pade approximations of $h^{\prime \prime \prime}(0), f^{\prime}(0)$ and $g^{\prime}(0)$ when $R=10$.

\begin{tabular}{|c|c|c|c|}
\hline$[m, m]$ & $h^{\prime \prime \prime}(0)$ & $f^{\prime}(0)$ & $g^{\prime}(0)$ \\
\hline$[5,5]$ & -38.1021 & -3.46043 & -2.40875 \\
\hline$[10,10]$ & -38.1024 & -3.46051 & -2.40862 \\
\hline$[15,15]$ & -38.1024 & -3.46051 & -2.40862 \\
\hline$[20,20]$ & -38.1024 & -3.46051 & -2.40862 \\
\hline
\end{tabular}

The results for velocity profiles and lift and drag coefficients have been presented in the form of graphs and Tables. The effects of the cross flow Reynolds number $R$ on the velocity components of $h, f$ and $g$ are shown in Figures 3-5.

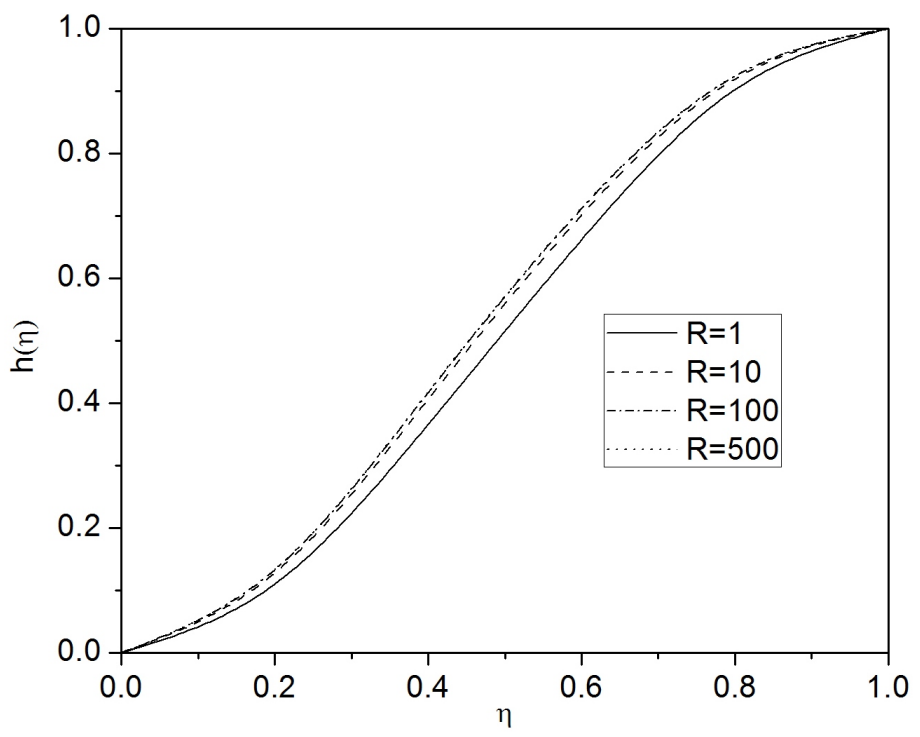

Fig. 3 Variations of $h$ by increasing Reynolds number $R$. 
From the Fig. 3, it is observed that the velocity profiles of $h$ increases with the increasing values of $R$. The influence of $R$ on $f$ is described in Fig. 4, it shows that velocity profiles are decreasing with increasing values of $R$. The effect of $R$ on velocity components of $g$ are shown in Fig. 5. Here, the velocity profile depicts the same behaviour as compared to $f$.

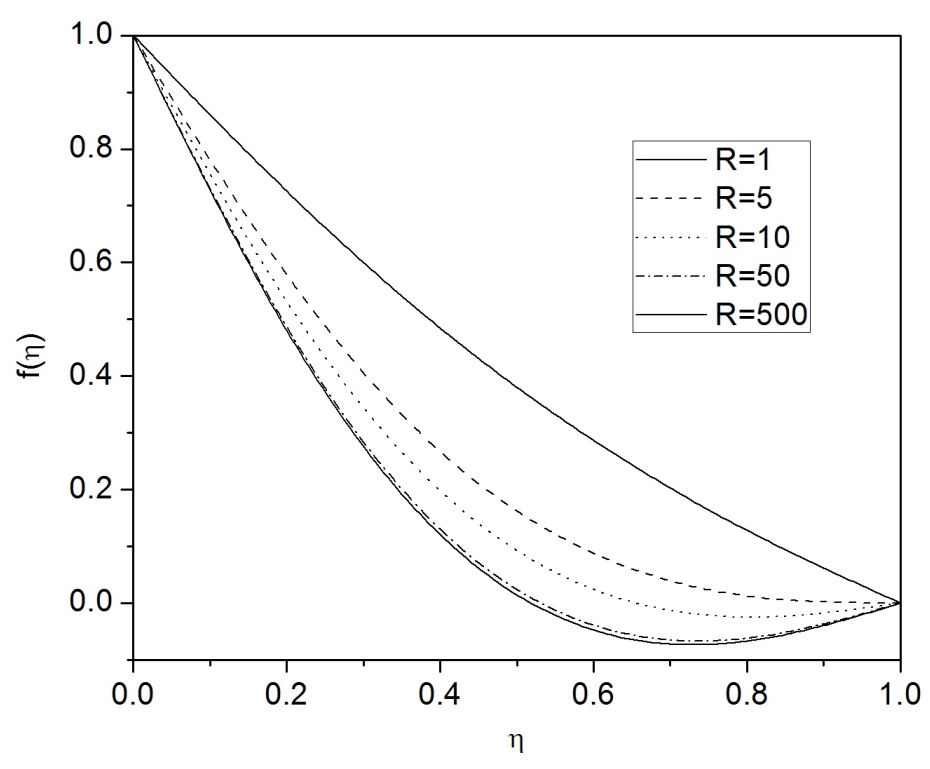

Fig. 4 Variations of $f$ by increasing Reynolds number $R$.

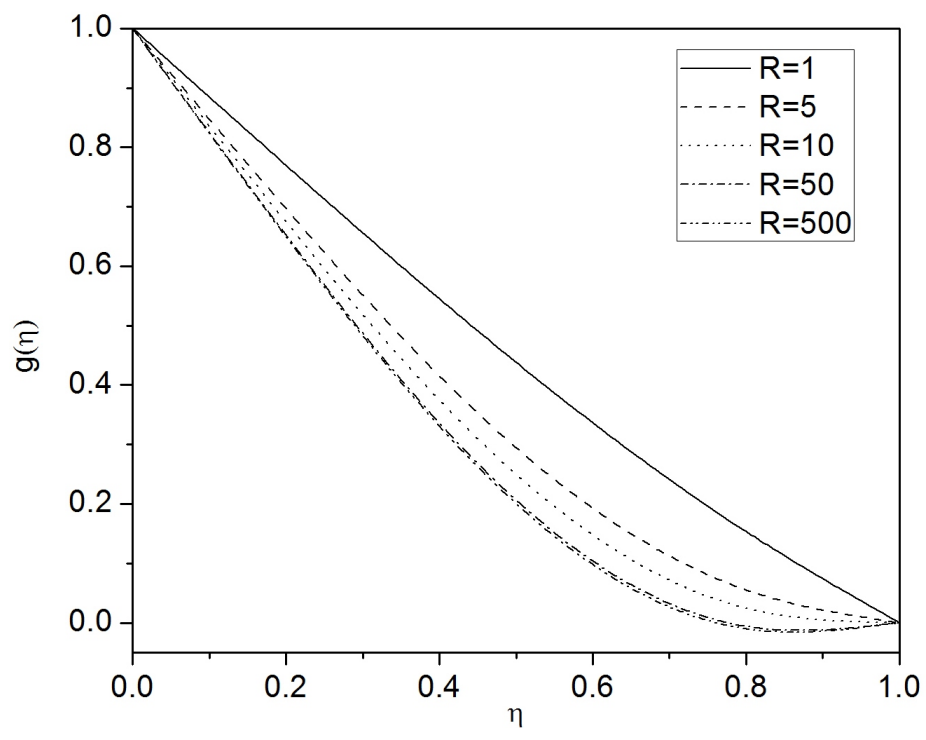

Fig. 5 Variations of $g$ by increasing Reynolds number $R$.

The lift and drag can be calculated by the following relations:

$$
\begin{array}{ll}
L=\left(\frac{\rho W^{4} L_{1} L_{2}}{12 \gamma^{2}}\right)\left(\frac{-h^{\prime \prime \prime}(0)}{R^{3}}\right) & \text { Lift } \\
D_{x}=\rho U W L_{1} L_{2}\left(\frac{-f^{\prime}(1)}{R}\right) & \text { Drag in } x \text {-direction }
\end{array}
$$




$$
D_{y}=\rho U W L_{1} L_{2}\left(\frac{-g^{\prime}(1)}{R}\right) \quad \text { Drag in } y \text {-direction }
$$

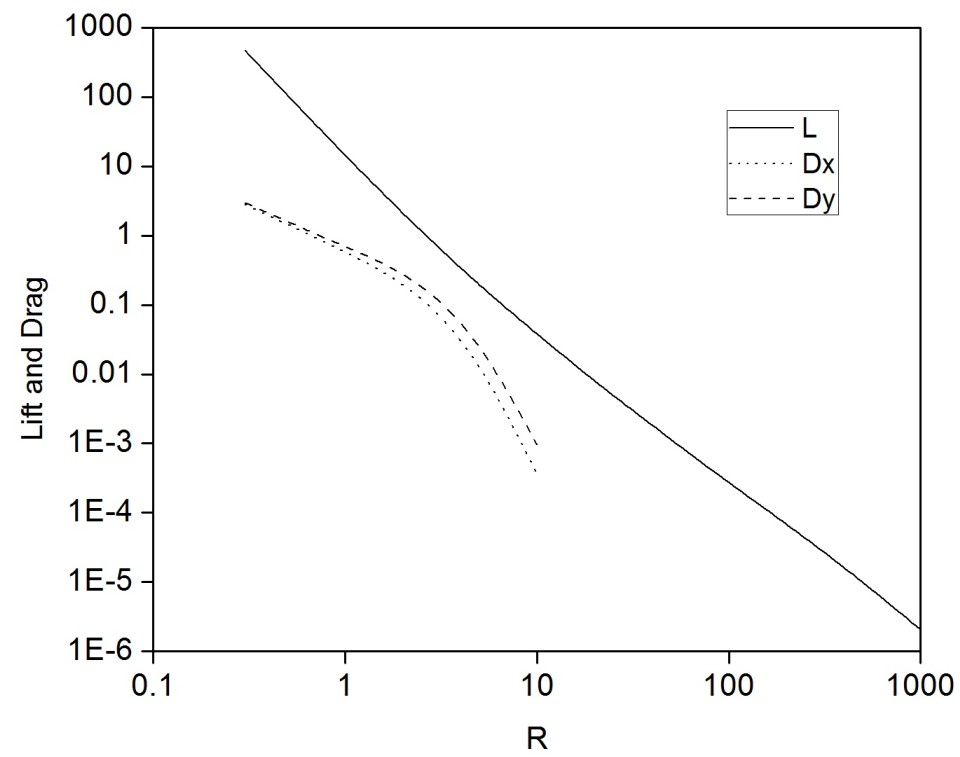

Fig. 6 Normalized lift and drag as a function of Reynolds number.

Fig. 6 shows the normalized lift $(L)$ and drag coefficients $\left(D_{x}\right.$ and $\left.D_{y}\right)$ as a function of $R$, it is seen that the magnitudes of the lift and drag were decreased with the increase in Reynolds number. In order to see the variations of $h^{\prime \prime \prime}(0), f^{\prime}(0)$ and $g^{\prime}(0)$ for different values of Reynolds number are given in Table 3.

Table 3 Comparison of the Homotopy pade approximations (HPA) [40, 40] solution with the numerical solution [2] and long series (LS) [16] for different values of the Reynolds number $R$.

\begin{tabular}{|c|c|c|c|c|c|c|c|c|c|}
\hline & & $h^{\prime \prime \prime}(0)$ & & & $f^{\prime}(0)$ & & & $g^{\prime}(0)$ & \\
\hline $\mathrm{R}$ & HPA & Numerical & LS & HPA & Numerical & LS & HPA & Numerical & LS \\
\hline 0.2 & -12.465 & -12.465 & -12.447 & -1.088 & -1.088 & -1.085 & -1.030 & -1.030 & -1.030 \\
\hline 1 & -14.365 & -14.365 & -14.196 & -1.405 & -1.405 & -1.406 & -1.153 & -1.153 & -1.165 \\
\hline 5 & -24.583 & -24.584 & -22.893 & -2.527 & -2.528 & -2.991 & -1.766 & -1.766 & -1.859 \\
\hline 13.8 & -48.480 & -48.484 & -48.068 & -4.021 & -4.022 & -4.224 & -2.806 & ---- & -2.807 \\
\hline 51.6 & -149.67 & -149.67 & ---- & -7.553 & -7.553 & --- & -5.302 & -5.301 & --- \\
\hline 70 & -197.96 & ---- & ---- & -8.757 & ---- & --- & -6.144 & ---- & --- \\
\hline 100 & -275.90 & ---- & ---- & -10.40 & ---- & --- & -7.288 & ---- & --- \\
\hline 300 & -788.43 & ---- & ---- & -17.815 & ---- & --- & -12.516 & ---- & --- \\
\hline 500 & -1291.1 & ---- & ---- & -22.670 & ---- & --- & -15.368 & ---- & --- \\
\hline 1000 & -2526.4 & ---- & ---- & -30.432 & ---- & --- & -20.062 & ---- & --- \\
\hline
\end{tabular}

It is observed that the values of $h^{\prime \prime \prime}(0), f^{\prime}(0)$ and $g^{\prime}(0)$ decreases with increasing Reynolds number. From the Table 3, we have seen that the HAM results agree very well and have good accuracy as compared to previous literature results [2]- [16]. Also the HAM results are converges for much larger Reynolds number as compared to HPM, ADM and numerical results. 


\section{Conclusion}

In this article, we describe the analysis of coupled boundary value problem for eighth order nonlinear ordinary differential equation over finite interval arising in three dimensional flow problems. The semi-analytical schemes described here offer advantages over solutions obtained by long series, HPM, ADM and other numerical methods etc. The convergence of the Homotopy analysis method is given. The results are presented in the form of Tables and graphs, also the effects of cross flow Reynolds number is discussed for much larger domain.

\section{References}

[1] C.Y. Wang, (1974), Fluid Dynamics of the Circular Porous Slider, Journal of Applied Mechanics, 41, pp 343-347.

[2] F.M. Shalak and C.Y. Wang, (1975), Fluid Dynamics of a Long Porous Slider, Journal of Applied Mechanics, 42, pp 893-894. doi 10.1115/1.3423733

[3] F.M. Shalak and C.Y. Wang, (1978), On the Non-unique Solutions of Laminar Flow through a Porous Tube or Channel, SIAM Journal on Applied Mathematics, 34, pp 535-544. doi 10.1137/0134042

[4] C.Y. Wang, (1978), The Elliptic Porous Slider at Low Crossflow Reynolds Numbers, Journal of Lubrication Technology, 100, pp 444-446. doi 10.1115/1.3453206

[5] L.T. Watson, T.Y. Li and C.Y. Wang, (1978), Fluid Dynamics of the Elliptic Porous Slider, Journal of Applied Mechanics, 45, pp 435-436. doi 10.1115/1.3424320

[6] A.S. Berman, (1953), Laminar Flow in a Channel with Porous Walls, Journal of Applied Physics, 24, pp 1232-1235. doi 10.1063/1.1721476

[7] I. Proudman, (1960), An Example of Study Laminar Flow at large Reynolds Number, Journal of Applied Mechanics, 9, pp 593-602. doi 10.1017/S002211206000133X

[8] R.M. Terrill, (1964), Laminar Flow in a Uniformly Porous Channel, Aeronautical Quarterly, 15, pp 299-310.

[9] A.F. Elkouh, (1968), Laminar Flow between Rotating Porous Disks, Journal of the Engineering Mechanics Division, 94, pp 919-929.

[10] H. Rasmussen, (1970), Steady Viscous Flow between Two Porous Disks, Zeitschrift fur Angewandte Mathematik und Physik, 21, pp 187-195. doi 10.1007/BF01590643

[11] J. F. Brady, (1984), Flow Development in a Porous Channel and Tube, Physics of Fluids, 27, pp 1061-1067. doi $10.1063 / 1.864735$

[12] Vishwanath. B. Awati, Manjunath Jyoti and N. N. Katagi, (2016), Computer extended series and Homotopy analysis method for the solution of MHD flow of viscous fluid between two parallel porous plates, Gulf Journal of Mathematics, 4, 65-79.

[13] S.J. Liao, (1992), On the proposed homotopy analysis techniques for nonlinear problems and its application, Ph.D Dissertation, Shanghai Jiao Tong University.

[14] S.J. Liao, (2003), Beyond perturbation: An introduction to the homotopy analysis method, London/Boca Raton: Chapman \& Hall/CRC; Press.

[15] S.J. Liao, (2009), Notes on the homotopy analysis method: some definitions and theorems, Commun. Nonlinear. Sci. Numer. Simulat., 14:983. doi 10.1016/j.cnsns.2008.04.013

[16] V.S. Madalli, N.M. Bujurke and B.G. Mulimani, (1995), Lubrication of a long porous slider, Tribology International, 28: 4, pp225-232. doi 10.1016/0301-679X(94)00006 
(OUP4 Sciences. All rights reserved. 